Research Paper

\title{
Anal adenocarcinoma requires prophylactic inguinal nodal treatment: Results from a single Chinese institution
}

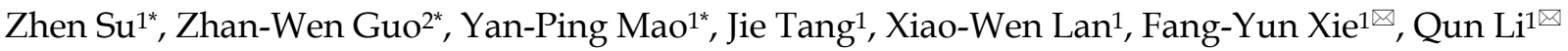 \\ 1. Department of Radiation Oncology, Sun Yat-Sen University Cancer Center; State Key Laboratory of Oncology in South China; Collaborative Innovation \\ Center for Cancer Medicine, Guangzhou 510060, China; \\ 2. Department of Radiation Oncology, The General Hospital of Shenyang Military Area Command, No.83, Wenhua Road, Shenhe District, Shenyang, Liaoning \\ province, 110016, China. \\ * These authors contributed equally to this work. \\ $\triangle$ Corresponding authors: Phone: +86 02087343029. E-mail: liqun@sysucc.org.cn. Phone: +86 02087343484. E-mail: xiefy0758@sina.com.
}

(c) Ivyspring International Publisher. This is an open access article distributed under the terms of the Creative Commons Attribution (CC BY-NC) license (https://creativecommons.org/licenses/by-nc/4.0/). See http://ivyspring.com/terms for full terms and conditions.

Received: 2016.09.08; Accepted: 2016.12.23; Published: 2017.04.09

\begin{abstract}
Background: Literature pertaining to prophylactic inguinal nodal treatment for anal adenocarcinoma in China is scarce.

Methods: In this retrospective study, we analyzed 126 patients from 1965 to 2015 . Among these, 67 patients received surgery only, 18 patients received chemoradiotherapy only, 27 patients received a combination of both, and the remaining 14 patients received palliative treatment.

Results: The median follow up period was 30 months. The 1-year, 3-year, and 5-year overall survival rates were $85.8 \%, 62.5 \%$, and $43.4 \%$, respectively. The 5 -year overall survival was $46.9 \%$ for patients with negative inguinal lymph nodes and $19.1 \%$ for patients with positive inguinal lymph nodes $(p=0.007)$. The overall 5 -year inguinal node relapse-free survival was $83.0 \%$. The 5 -year inguinal node relapse-free survival was $87.5 \%$ for stage I, $86.9 \%$ for stage II, and $76.5 \%$ for stage III cancers. Among those with negative inguinal nodes, the 5-year inguinal node relapse-free survival was $85.7 \%$ for negative regional lymph nodes and $75.4 \%$ for positive regional lymph nodes $(p=0.089)$.

Conclusion: Inguinal lymph node is a high-risk subclinical area. Prophylactic inguinal nodal treatment is necessary for patients with anal adenocarcinoma irrespective of positive or negative inguinal lymph nodes.
\end{abstract}

Key words: Anal adenocarcinoma; inguinal lymph node; prophylactic inguinal nodal treatment; overall survival; inguinal node relapse-free survival.

\section{Background}

Anal carcinoma is a rare type of cancer, which accounts for approximately $1.5 \%-2.5 \%$ of all digestive system cancers $[1,2]$. However, the worldwide incidence rate has steadily increased: In the United States of America, for example, it increased by approximately 1.9-fold among men and 1.5-fold among women from the period of 1973 through 1979 to 1994 through 2000, respectively, and has continued to increase ever since. Most primary cancers of the anal canal are squamous cell cancers [3, 4]. The recommended first-line treatment for patients with stage II/III anal carcinoma is concurrent chemoradiotherapy with mitomycin-C and 5-fluorouracil (5-Fu) [5-8]. While initially, the radiation fields did include the inguinal nodes and the pelvis, anus, and perineum $[9,10]$, the need for inclusion of inguinal nodes in the radiation fields is still inconclusive. Several randomized trials have 
shown that elective irradiation of the groin should be considered for all tumors to reduce inguinal progression risk [6, 11-13]. A few other reports suggested that prophylactic inguinal nodal irradiation (PINI) should be omitted to reduce the radiation field size without compromising on locoregional control for selected anal cancer patients [14, 15]. On the other hand, with the longer-term follow up, patients and doctors pay more attention to the quality of life after treatment and increase concerns about late complications such as anal ulcers, stenosis necrosis, and higher risk of subsequent pelvic fracture [16-19].

Furthermore, it must be noted that the existing National Comprehensive Cancer Network (NCCN) guidelines are not always suitable for Chinese patients, because the guidelines are based on data from European and American patient populations. Interestingly, the most common pathology of anal carcinomas in China is adenocarcinoma, which is different from that in European and American populations. Therefore, there is insufficient data regarding the necessity of inguinal lymph node involvement in radiation targets, among Chinese patients.

We present herein a retrospective study to analyze the patterns of failure in anal cancer patients who underwent treatment at our institution to evaluate the necessity for prophylactic inguinal nodal treatment and provide evidence for clinical practice from China, where adenocarcinoma are the most common form of anal cancers.

\section{Methods}

\section{Patients}

We reviewed the records of 200 patients with anal cancer who underwent treatment at the Sun Yat-Sen University Cancer Centre between January 1965 and September 2015. Among these, 74 patients were excluded from the study because of biopsy-proven melanoma (17 patients) or squamous cell carcinoma (36 patients), unknown TNM or pathology (16 patients), and follow up less than 3 months (5 patients). Finally, 126 patients with biopsy-proven anal adenocarcinoma with no distant metastases at diagnosis were included for analysis. All tumors were restaged based on the seventh edition of the American Joint Committee on Cancer/Union for International Cancer Control (AJCC/UICC) tumors staging criteria [20]. Tumor assessment before treatment included digital rectal examination (DRE), colonoscopy with biopsy, chest radiography, and abdominopelvic computed tomography (AP-CT) or abdominal ultrasound. Pelvic magnetic resonance imaging (MRI) or transanal/rectal ultrasound was optional. Of the 126 patients, 21 were diagnosed as having inguinal nodal metastasis by either image examination or biopsy confirmation.

\section{Treatment}

In all, 53.2\% (67/126) patients received abdominoperineal resection (APR) or tumorectomy (surgery alone), $14.3 \% \quad(18 / 126)$ received chemoradiotherapy, and $21.4 \%$ (27/126) received surgery in combination with radiotherapy or chemotherapy (comprehensive therapy). The other 14 patients $(11.10 \%)$ received palliative treatment.

Chemotherapy regimens and administering schedules showed some heterogeneity. All chemotherapy schedules were either 5-Fu-based mono-agent or multi-agent therapies. Most of the patients received a continuous infusion of 5-Fu (1000 $\mathrm{mg} / \mathrm{m}^{2}$ daily on days $\left.1-4\right)$ or capecitabine, or capecitabine plus oxaliplatin.

28 patients received radiotherapy (12 patients with 2 dimensional conformal radiation therapy, 3 patients with 3 dimensional conformal radiation therapy, and 13 patients with intensity-modulated radiation therapy). The prescribed dose was 46-60 Gy to the planning target volume (PTV1) of the GTV (primary gross tumor volume), and 46-50 Gy to the PTV2 of CTV (clinical target volume) in 23-30 fractions.

\section{Follow up and statistical analysis}

After treatment completion, the patients were followed up every 3 months for the first 3 years, every 6 months for the next 2 years, and then annually. The following endpoints (time to the first defined event) were assessed: overall survival (OS), locoregional relapse-free survival (LRFS), distant metastasis-free survival (DMFS), and inguinal node relapse-free survival (INRFS). OS was defined as the time from the date initially diagnosed to the date of death resulting from any cause. LRFS and DMFS were defined as the time from the date initially diagnosed to the date of first locoregional relapse and distant metastasis, respectively. INRFS was defined as the time from the date initially diagnosed to the date of inguinal node relapse.

Survival rates were evaluated using Kaplan-Meier curves and compared using the log-rank test. All statistical tests were two-sided, and a $p$ value $<0.05$ was considered statistically significant. All tests were performed using IBM SPSS 20.0.

\section{Results}

\section{Patient characteristics}

In this retrospective study, a total of 126 eligible 
patients were identified from 1965 to 2015. Table 1 shows the baseline clinical and treatment characteristics of patients. The male to female ratio was approximately 1.21:1. The median age of the population was 55.5 years (range, 17-89 years). In all, $74.6 \%$ (94/126) patients received APR or tumorectomy.

Table 1. Baseline clinical and treatment characteristics of patients $(n=126)$

\begin{tabular}{ll}
\hline Variable & No. $(\%)$ \\
\hline Age, median (range) & $55.5(17-89)$ \\
Sex & \\
Male & $69(54.8)$ \\
Female & $57(45.2)$ \\
Smoking & \\
No & $95(75.4)$ \\
yes & $31(24.6)$ \\
Grade & \\
High & $22(17.5)$ \\
Middle & $80(63.5)$ \\
low & $24(19.0)$ \\
Clinical tumor stage & \\
T1 & $9(7.1)$ \\
T2 & $49(38.9)$ \\
T3 & $55(43.7)$ \\
T4 & $13(10.3)$ \\
Clinical nodal stage & \\
N0 & $72(57.1)$ \\
N1 & $29(23.0)$ \\
N2 & $15(11.9)$ \\
N3 & $10(7.9)$ \\
TNM Stage & \\
I & $8(6.3)$ \\
II & $57(45.2)$ \\
III & $61(48.4)$ \\
Inguinal lymph nodes & \\
No & $105(83.3)$ \\
Treatment & $21(16.7)$ \\
Palliative treatment & \\
Chemoradiotherapy & $14(11.1)$ \\
Comprehensive therapy & $67(53.2)$ \\
& $18(14.3)$ \\
\hline & $27(21.4)$ \\
\hline
\end{tabular}

\section{Patterns of treatment failure and overall survival}

Up to the last day of follow up, 36 patients developed locoregional relapse, 25 patients developed distant metastasis and 75 patients died. The sites of locoregional relapse included local relapse (the rectum, anus, and presacral tissues) in 16 patients, regional nodes in 13 patients, and local relapse plus pelvic nodes in 7 patients.

With a median follow up of 30.0 months (range, 3.32-266.81 months), the 1-year, 3-year, and 5-year OS was $85.8 \%, 62.5 \%$, and $43.4 \%$, respectively (Figure 1A). The 1-year, 3-year, and 5-year LRFS was $89.0 \%$, $69.3 \%$, and $64.5 \%$, respectively (Figure 1B). The 1-year, 3 -year, and 5-year DMFS was 92.3\%, 81.2\%, and $72.8 \%$, respectively (Figure $1 C$ ). The 5 -year OS was
$46.9 \%$ for patients with negative inguinal lymph nodes and $19.1 \%$ for patients with positive inguinal lymph $(p=0.007)$.

\section{Inguinal nodal recurrence}

Up to the last day of follow up, 17 patients $(13.7 \%)$ developed inguinal nodal recurrence. The 5 -year actuarial rate of inguinal recurrence was $17.0 \%$. The 1-year, 3-year, and 5-year INRFS was 93.1\%, $84.5 \%$, and $83.0 \%$, respectively (Figure 1D). The 5-year INRFS was $87.5 \%$ for stage I, $86.9 \%$ for stage II, and $76.5 \%$ for stage III cancers. The 5-year INRFS was $82.6 \%$ for patients with negative inguinal node and $89.5 \%$ for patients with positive inguinal node $(p=0.986)$. Among the patients with negative inguinal node, the 5-year INRFS was $85.7 \%$ for negative regional lymph nodes and $75.4 \%$ for positive regional lymph nodes $(p=0.089)$.

\section{Discussion}

In this study, we found that the 5-year inguinal recurrence rate in anal adenocarcinoma patients with stage I-III cancers was $17.0 \%$. The patients with positive inguinal lymph nodes had a poorer survival than those with negative inguinal lymph nodes. The inguinal lymph node treatment should hence be considered seriously.

It is well known that the American Joint Committee on Cancer/Union for International Cancer Control (AJCC/UICC) lymph node staging has distinct criteria such as the size, number, and location of regional lymph nodes [20]. Lymph node staging in anal canal cancer is based on the location of involved nodes, which is different from the lymph node staging in rectal cancer [20,21]. Lymphatic drainage in anal tumors is dependent on the location of the tumor in the anal region [20]. Lymph drainage at and proximal to the dentate line is directed toward the anorectal, perirectal, and paravertebral nodes and to some of the internal iliac system nodes [9]. Lymph drain of the lower anal canal and external anal sphincter is via the perianal plexuses into vessels then the external inguinal lymph nodes. Cancers in the perianal skin and the anal canal distal to the dentate line drain mainly to the superficial inguinal nodes [9]. However, considering this marked variation in the lymphatic drainage and the numerous connections between lymphatics at various levels of the anal canal [22], distal anal cancers present with a higher incidence of inguinal node metastasis. Proximal anal cancer can occur inguinal node metastases, because the lymphatic drainage systems throughout the anal canal are not isolated from each other. In our study, the incidence of inguinal node metastases at diagnosis was $22.6 \%$. The patients with positive inguinal lymph 
nodes had a poorer survival than those with negative inguinal lymph nodes $(19.1 \%$ vs. $46.9 \%$, respectively, $p=0.007$ ). In previous studies, the risk for subclinical disease in the inguinal lymph nodes ranged from $18 \%$ to $28 \%$ [23]. Therefore, we inferred that the inguinal lymph node is a high-risk region requiring significant clinical attention. In a series of patients with anal cancer who underwent an APR, it was noted that pelvic nodal metastasis were often under $0.5 \mathrm{~cm}$, suggesting that routine radiologic evaluation with computed tomography and positron emission tomography scan may not be reliable in the determination of lymph node involvement [24]. Therefore, regardless of the lymph node size, presence of inguinal lymph nodes should be treated with caution especially in patients with pelvic nodes.

According to the Radiation Therapy Oncology Group (RTOG) 0529 guideline, the initial radiation fields should include the inguinal nodes, pelvis, anus, and the perineum, and the lateral border should include the lateral inguinal nodes [10]. As per the Australasian Gastrointestinal Trials Group (AGITG) guidelines, all elective regions should be routinely contoured for all disease stages, with the possible exception of the inguinal and high pelvic nodes for select, early-stage T1N0 tumors [25]. Anal squamous cell cancers have regional lymph node spread to three sites-rectal mesenteric, hypogastric, and inguinal and all such regional sites should be included in the initial radiation treatment fields [26, 27]. Inguinal metastases were found in $32 \%$ of anal adenocarcinoma [28]. A report from the University of Florida stated that elective irradiation of the inguinal lymph nodes is highly effective in controlling subclinical disease from carcinomas originating in the pelvis. Further, the inguinal lymph node control rate was $96 \%$ and can be accomplished with minimal acute or long-term complications [23]. Two recent studies have shown that with radiation to elective node irradiation, excellent nodal control was achieved in the inguinal region, with control rates as high as $98.5 \%$ and $100.0 \%$ $[29,30]$. A French study showed that the 5-year cumulative rate of inguinal recurrence was $2 \%$ and $16 \%$ in prophylactic inguinal irradiation (PII) and no PII groups, respectively $(p=0.006)$. PII should be mandated for all T3-4 tumors. Moreover, PII should also be considered for T1-2 tumors, because the 5-year inguinal recurrence risk remains substantial when omitting PII (about 10\%) [12]. These studies showed that inguinal node radiation achieved excellent nodal control, and the inguinal node should be involved in the radiation field. Blinde et al. found that the 5-year inguinal recurrence rate was high in patients who did not receive elective irradiation therapy to the groin and suggested that elective irradiation of the groin should be considered for local control [31]. The results of the Trans-Tasman Radiation Oncology Group study (TROG) 99.02 also showed that T1-2 anal carcinomas require elective inguinal radiation treatment [32]. Our study found that the 5-year actuarial rate of inguinal recurrence was $17.0 \%$, which is consistent with previous reports [23]. Inguinal lymph node treatment is necessary for patients with anal carcinoma. It must be noted that in these studies, anal carcinoma pathology was squamous cell carcinoma, and the data were from small samples of European and American populations. In China, however, the main pathology of anal carcinoma is adenocarcinoma. There are only limited studies in this regard, especially the data concerning inguinal lymph node radiation for anal adenocarcinoma. To the best of our knowledge, our study is the first one to discuss the necessary treatment for inguinal lymph nodes in anal adenocarcinoma and recommend that inguinal lymph nodes should be involved in the radiation field or treated by surgery.

Inguinal lymph node involvement presents a poor prognosis for anal carcinoma. In our study, patients with positive inguinal lymph had a poorer survival than those with negative inguinal lymph $(19.1 \%$ vs. $46.9 \%, p=0.007)$. A report about management of inguinal lymph node metastases showed that the 5-year OS rate of patients with synchronous inguinal metastases was higher than those with metachronous inguinal metastases $(54.4 \%$ vs. $41.4 \%$ ) [33]. This difference in OS is likely because no elective irradiation of clinically normal inguinal areas was performed, but patients with metastatic inguinal lymph nodes were treated with inguinal dissection and postoperative irradiation with a dose of 50 Gy over 5 weeks. This indicates that inguinal lymph node treatment could improve survival rates in anal carcinoma.

However, some studies had conflicting results. A report from Switzerland indicated that the inguinal relapse rate remains relatively low with or without elective inguinal node radiation therapy (INRT) for patients with node-negative T2 anal cancer, for which the overall 5-year INRFS was 92.3\%. Furthermore, patients with INRT experienced a higher rate of grade $\geq 3$ acute toxicity than those without INRT (53\% vs. $31 \%, p=0.076$ ) [34]. This study concluded that the role of INRT in treatment of early-stage anal carcinoma needs further investigation. A Korean study also had similar conclusions, in that INRT omission should be considered for patients with negative inguinal nodes [35]. It is noteworthy that one of these studies had a small sample size $(n=33)$ and the other one only included patients with T2 staging, and the cancer type was squamous cell cancer in both [34, 35]. 

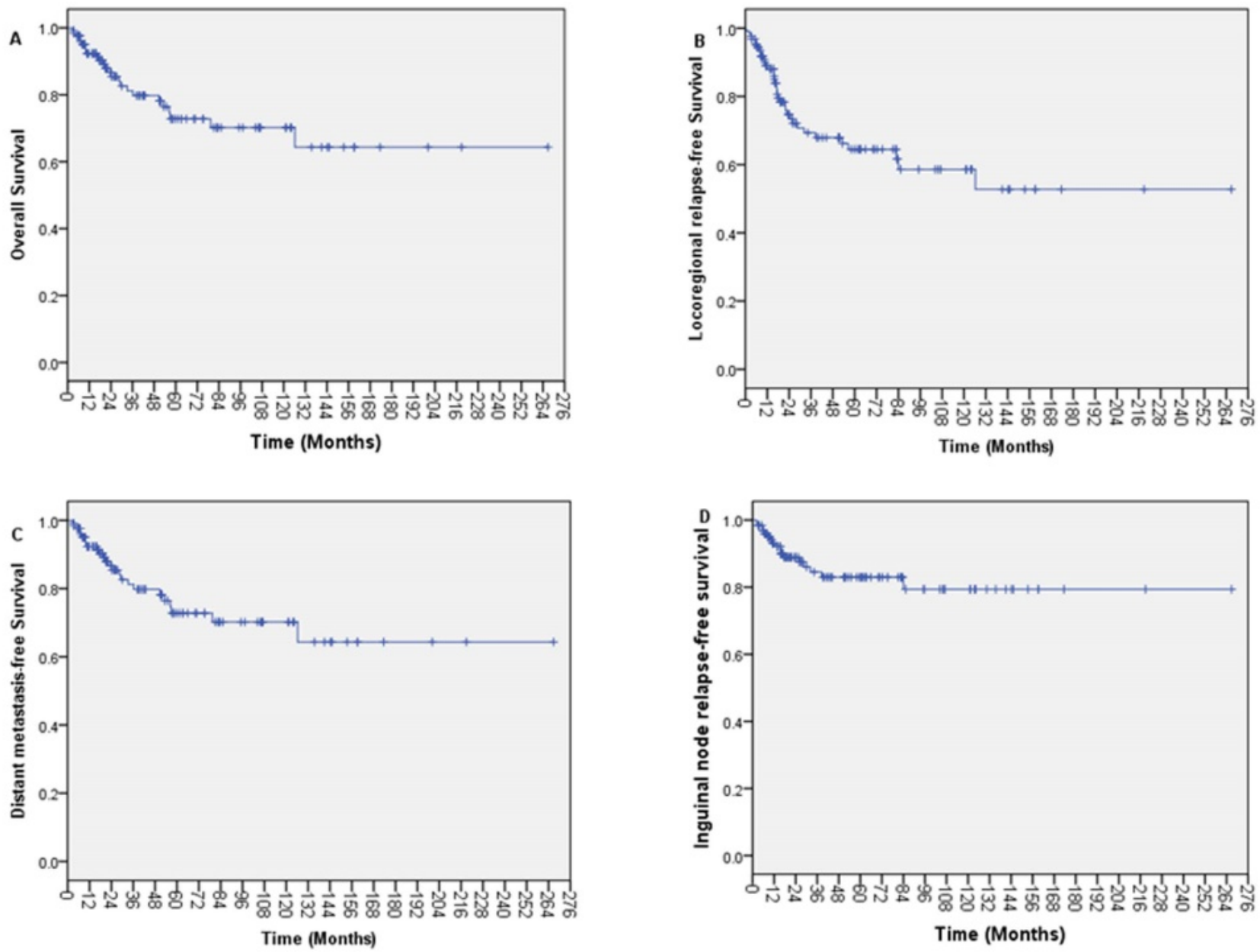

Figure 1. Kaplan-Meier survival curves for patients with anal adenocarcinoma. (A) Overall survival; (B) Locoregional relapse-free survival; (C) Distant metastasis-free survival and (D) Inguinal node relapse-free survival.

Furthermore, two studies about rectal adenocarcinoma extending to the anal canal presented similar conclusions, in that INRT was not necessary for rectal cancer with anal canal invasion owing to low inguinal lymph node recurrence rate $[36,37]$. The primary lymphatic drainage of the rectum is to the perirectal and pelvic lymph nodes [38]. Inguinal lymph node metastases from rectal adenocarcinoma are rare [39]. Although anal adenocarcinoma has the same pathology as rectal adenocarcinoma, they have different lymphatic drainage systems according to their different anatomical locations.

We acknowledge that our study has several limitations. First, this was a single-center retrospective analysis. Thus, prospective studies are needed to confirm our result. Second, the sample was small due to the rarity of the cancer and the median follow up was relatively short. Third, the treatment regimens varied among patients. Most of all, heterogeneous methods were used to evaluate inguinal node involvement. Further prospective studies are needed to confirm whether prophylactic inguinal nodal irradiation is necessary for patients with anal adenocarcinoma.

\section{Conclusion}

In conclusion, our findings indicate that the inguinal lymph node is a high-risk subclinical area. It is essential to consider and clinically evaluate the inguinal lymph nodes at diagnosis and obtain systematic imaging data of the inguinal lymphatics. We emphasize that prophylactic inguinal nodal treatment is necessary for patients with anal adenocarcinoma regardless of whether the inguinal lymph node is positive or negative.

\section{Abbreviations}

5-Fu: 5-fluorouracil; PINI: prophylactic inguinal nodal irradiation; NCCN: National Comprehensive Cancer Network; AJCC/UICC: American Joint Committee on Cancer/Union for International Cancer Control; DRE: digital rectal examination; AP-CT: abdominopelvic computed tomography; MRI: 
magnetic resonance imaging; APR: abdominoperineal resection; PTV: planning target volume; GTV: primary gross tumor volume; CTV: clinical target volume; OS: overall survival; LRFS: locoregional relapse-free survival; DMFS: distant metastasis-free survival; INRFS: inguinal node relapse-free survival; RTOG: Radiation Therapy Oncology Group; AGITG: Australasian Gastrointestinal Trials Group; PII: prophylactic inguinal irradiation; INRT: elective inguinal node radiation therapy.

\section{Acknowledgments}

We appreciate all individuals, particularly the people of Sun Yat-Sen University Cancer Center, including doctors, nurses, coordinators, and the Data Management team who generously spared their time for the accomplishment and fulfillment of this project.

\section{Ethics approval and consent to participate}

This study was reviewed and approved by the institutional review board and ethics committee of Sun Yat-Sen University Cancer Center. All patient records were anonymized and de-identified prior to analysis.

\section{Competing Interests}

The authors have declared that no competing interest exists.

\section{References}

1. Siegel R, Ma J, Zou Z, et al. Cancer statistics, 2014. CA: A Cancer Journal for Clinicians. 2014; 64: 9-29.

2. Whiteford MH, Jr Stevens K, Oh S, et al. The evolving treatment of anal cancer: How are we doing? Arch Surg. 2001; 136: 886-91.

3. Nelson RA, Levine AM, Bernstein L, et al. Changing patterns of anal canal carcinoma in the United States. J Clin Oncol. 2013; 31: 1569-75.

4. Jemal A, Simard E P, Dorell C, et al. Annual Report to the Nation on the Status of Cancer, 1975-2009, featuring the burden and trends in human papillomavirus(HPV)-associated cancers and HPV vaccination coverage levels. J Natl Cancer Inst. 2013;105: 175-201.

5. Bartelink H, Roelofsen F, Eschwege F, et al. Concomitant radiotherapy and chemotherapy is superior to radiotherapy alone in the treatment of locally advanced anal cancer: results of a phase III randomized trial of the European Organization for Research and Treatment of Cancer Radiotherapy and Gastrointestinal Cooperative Groups. J Clin Oncol. 1997; 15: 2040-2049.

6. Flam M, John M, Pajak T F, et al. Role of mitomycin in combination with fluorouracil and radiotherapy, and of salvage chemoradiation in the definitive nonsurgical treatment of epidermoid carcinoma of the anal canal: results of a phase III randomized intergroup study. J Clin Oncol. 1996; 14: 2527-2539.

7. [No authors listed]. Epidermoid anal cancer: results from the UKCCCR randomised trial of radiotherapy alone versus radiotherapy, 5-fluorouracil, and mitomycin. UKCCCR Anal Cancer Trial Working Party. UK Co-ordinating Committee on Cancer Research. Lancet. 1996;348: 1049-1054.

8. Cummings B J, Keane T J, O'Sullivan B, et al. Epidermoid anal cancer: treatment by radiation alone or by radiation and 5-fluorouracil with and without mitomycin C. Int J Radiat Oncol Biol Phys. 1991; 21: 1115-1125.

9. 9. Paul F. Engstrom, Juan Pablo Arnoletti, Al B. Benson III, et al. NCCN clinical practice guidelines in oncology. Anal carcinoma. J Natl Compr Canc Netw. $2010 ; 8: 106-20$.

10. Myerson R J, Garofalo M C, El N I, et al. Elective clinical target volumes for conformal therapy in anorectal cancer: a radiation therapy oncology group consensus panel contouring atlas. Int J Radiat Oncol Biol Phys. 2009; 74: 824-830.

11. James R D, Glynne-Jones R, Meadows H M, et al. Mitomycin or cisplatin chemoradiation with or without maintenance chemotherapy for treatment of squamous-cell carcinoma of the anus (ACT II): a randomised, phase 3, open-label, 2 × 2 factorial trial. Lancet Oncol. 2013; 14: 516-524
12. Ortholan $C$, Resbeut $M$, Hannoun-Levi J M, et al. Anal canal cancer: management of inguinal nodes and benefit of prophylactic inguinal irradiation (CORS-03 Study). Int J Radiat Oncol Biol Phys. 2012; 82: 1988-1995.

13. Ajani J A, Winter K A, Gunderson L L, et al. Fluorouracil, mitomycin, and radiotherapy vs fluorouracil, cisplatin, and radiotherapy for carcinoma of the anal canal: a randomized controlled trial. JAMA. 2008; 299: 1914-1921.

14. Crowley C, Winship A Z, Hawkins M A, et al. Size does matter: can we reduce the radiotherapy field size for selected cases of anal canal cancer undergoing chemoradiation? Clin Oncol (R Coll Radiol). 2009; 21: 376-379.

15. Bartelink H, Roelofsen F, Eschwege F, et al. Concomitant radiotherapy and chemotherapy is superior to radiotherapy alone in the treatment of locally advanced anal cancer: results of a phase III randomized trial of the European Organization for Research and Treatment of Cancer Radiotherapy and Gastrointestinal Cooperative Groups. J Clin Oncol. 1997; 15: 2040-2049.

16. Das P, Cantor S B, Parker C L, et al. Long-term quality of life after radiotherapy for the treatment of anal cancer. Cancer. 2010; 116: 822-829.

17. De Bree E, Van Ruth S, Dewit L G, et al. High risk of colostomy with primary radiotherapy for anal cancer. Ann Surg Oncol. 2007; 14: 100-108.

18. Baxter N N, Habermann E B, Tepper J E, et al. Risk of pelvic fractures in older women following pelvic irradiation. JAMA. 2005; 294: 2587-2593.

19. Allal A S, Sprangers M A, Laurencet F, et al. Assessment of long-term quality of life in patients with anal carcinomas treated by radiotherapy with or without chemotherapy. Br J Cancer. 1999; 80: 1588-1594.

20. Edge S B, Compton C C. The American Joint Committee on Cancer: the 7th edition of the AJCC cancer staging manual and the future of TNM. Ann Surg Oncol. 2010; 17: 1471-1474.

21. Licitra L, Spinazze S, Doci R, et al. Cancer of the anal region. Crit Rev Oncol Hematol. 2002; 43: 77-92.

22. Lengele $B$, Scalliet P. Anatomical bases for the radiological delineation of lymph node areas. Part III: Pelvis and lower limbs. Radiother Oncol. 2009; 92: 22-33.

23. Lee W R, Mccollough W M, Mendenhall W M, et al. Elective inguinal lymph node irradiation for pelvic carcinomas. The University of Florida experience. Cancer. 1993;72: 2058-2065.

24. Wade D S, Herrera L, Castillo N B, et al. Metastases to the lymph nodes in epidermoid carcinoma of the anal canal studied by a clearing technique. Surg Gynecol Obstet. 1989; 169: 238-242.

25. Ng M, Leong T, Chander S, et al. Australasian Gastrointestinal Trials Group (AGITG) Contouring Atlas and Planning Guidelines for Intensity-Modulated Radiotherapy in Anal Cancer. International Journal of Radiation Oncology*Biology*Physics. 2012; 83: 1455-1462.

26. Cohen A M, Wong W D. Anal squamous cell cancer nodal metastases: prognostic significance and therapeutic considerations. Surg Oncol Clin N Am. 1996; 5: 203-210.

27. Henderson R H, Parsons J T, Morgan L, et al. Elective ilioinguinal lymph node irradiation. Int J Radiat Oncol Biol Phys. 1984; 10: 811-819.

28. Shindo K. Epidermoid carcinoma compared with adenocarcinoma of the anal canal. Aust N Z J Surg. 1981; 51: 426-429.

29. Henkenberens C, Meinecke D, Michael S, et al. Reduced radiation dose for elective nodal irradiation in node-negative anal cancer: back to the roots? Strahlenther Onkol. 2015; 191: 845-854.

30. Lepinoy A, Lescut N, Puyraveau M, et al. Evaluation of a 36Gy elective node irradiation dose in anal cancer. Radiother Oncol. 2015; 116: 197-201.

31. Blinde S E, Schasfoort R, Mens J W, et al. Inguinal lymph node recurrence in the untreated groin of patients with anal carcinoma. Dis Colon Rectum. 2014; 57: 578-584.

32. Matthews J H L, Burmeister B H, Borg M, et al. T1-2 anal carcinoma requires elective inguinal radiation treatment-The results of Trans Tasman Radiation Oncology Group study TROG 99.02. Radiotherapy and Oncology. 2011; 98: 93-98

33. Gerard J P, Chapet O, Samiei F, et al. Management of inguinal lymph node metastases in patients with carcinoma of the anal canal: experience in a series of 270 patients treated in Lyon and review of the literature. Cancer. 2001; 92: $77-84$.

34. Zilli $\mathrm{T}$, Betz $\mathrm{M}$, Bieri $\mathrm{S}$, et al. Elective inguinal node irradiation in early-stage T2N0 anal cancer: prognostic impact on locoregional control. Int J Radiat Oncol Biol Phys. 2013; 87: 60-66.

35. Kim H, Park H C, Yu J I, et al. Can we omit prophylactic inguinal nodal irradiation in anal cancer patients? Radiation Oncology Journal. 2015; 33: 83.

36. Yeo S G, Lim H W, Kim D Y, et al. Is elective inguinal radiotherapy necessary for locally advanced rectal adenocarcinoma invading anal canal? Radiat Oncol. 2014; 9: 296

37. Taylor N, Crane C, Skibber J, et al. Elective groin irradiation is not indicated for patients with adenocarcinoma of the rectum extending to the anal canal. Int J Radiat Oncol Biol Phys. 2001; 51: 741-747.

38. Hojo K, Koyama Y, Moriya Y. Lymphatic spread and its prognostic value in patients with rectal cancer. Am J Surg. 1982; 144: 350-354.

39. Mesko $\mathrm{T}$ W, Rodriguez-Bigas $\mathrm{M} \mathrm{A}$, Petrelli $\mathrm{N} \mathrm{J}$. Inguinal lymph node metastases from adenocarcinoma of the rectum. Am J Surg. 1994; 168: 285-287. 